\title{
EXPERIMENTS WITH BUNCH TRAINS IN LEP
}

\author{
O. Brunner, W. Herr, G. von Holtey, E. Keil, M. Lamont, M. Meddahi, J. Poole, R. Schmidt, A. Verdier, \\ C. Zhang, CERN, Geneva, Switzerland
}

Abstract

During 1994 tests were made to evaluate the possibility of operating LEP with four equidistant bunch trains in each beam during 1995. A train will consist of up to four bunches with a bunch spacing of some $75 \mathrm{~m}$. The bunch trains collide head-on. They are separated at the parasitic collision points on either side of the interaction points by vertical electrostatic separator bumps. The accumulation of bunch trains in a single beam was studied with magnetically simulated bumps. Following the installation of additional separators late in 1994, the parasitic encounters of the bunch trains near the interaction points were studied for one train of electron bunches colliding with one train of positron bunches in two diametrically opposite interaction points. The higherorder mode losses in the superconducting RF cavities were measured for several arrangements of beams, trains and bunches. The synchrotron radiation background, enhanced by the off-centred beams in the quadrupoles inside the separator bumps, is reduced by super-imposing asymmetric magnetic bumps such that the offset of the incoming beams is reduced.

\section{INTRODUCTION}

Bunch trains have been considered for the CESR storage ring at Cornell University since 1990 [1], and are now used in operation [2]. Thereafter, bunch trains with a horizontal crossing angle were proposed for LEP [3]. Tests in 1993 revealed insurmountable difficulties with the synchrotron radiation background, caused by the off-centred beams in the quadrupoles next to the LEP detectors [4]. A proposal [5] which overcomes this particular difficulty is described and the results of tests in 1994 are presented. The main changes are an increase of the bunch spacing and a vertical separation scheme which starts beyond the quadrupoles next to the LEP detectors. The orbital effects and background were studied with magnetic bumps, using vertical correctors. With up to four trains in a single beam, possible bunch current limitations, due to collective effects and higherorder mode losses in superconducting RF cavities were investigated. During several technical stops, the extra vertical electrostatic separators were installed [6]. Tests with colliding bunch trains are described and the conclusions are given.

A Bunch Train in LEP consists of up to four bunches. The spacing between bunches, about $s=74 \mathrm{~m}$, is much smaller than the spacing between trains. During tests in 1994, one train of electron bunches was collided with one train of positron bunches in two diametrically opposite interaction points, IP4 and IP8. The bunches were vertically separated at the three parasitic collision points near by, on either side.

\section{CONCEPT}

With the equipment installed for the tests, one bunch train in each beam could be used. The number of bunches within the trains and their spacing is determined by the requirement for sufficient separation at all parasitic encounters and the constraint from the LEP experiments that trains should not exceed a total length of $750 \mathrm{~ns}$ [7]. This spacing must be a multiple of $\lambda_{\mathrm{rf}}$ and the final choice was a spacing of $87 \lambda_{\text {rf }}$.

\section{A. Separator bumps}

The separator bumps in the even insertions allow the beams to be separated over the whole length of the bunch train during injection, ramping and squeezing as well as bringing them into collision at the interaction point while keeping the beams separated at the parasitic encounters.

In order to minimize the necessary changes, all existing vertical separators and 4 new ones were used [6]. At each experimental interaction point six separators provide a symmetric electrostatic bump over a length of about $240 \mathrm{~m}$ (Figure 1).

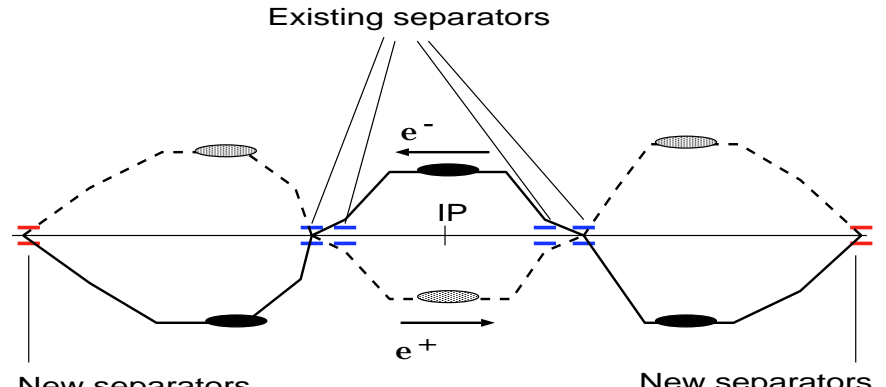

New separators

Figure. 1. Separation bump in the even points at injection, full line shows positron orbit and dashed line electrons. The two inner separator pairs are used to cancel the separation for physics.

\section{B. Side effects}

The amplitude of the electrostatic separator bumps should be large enough to minimize the effects caused by the parasitic beam-beam collisions, i.e. the beam-beam kicks, the beam-beam tune shifts, and their consequences. The bump amplitude should be small to minimize the vertical dispersion $D_{y}$ and the resulting vertical beam emittance $\epsilon_{y}$. Practically all these effects also depend on the direction of the bumps in IP4 and IP8 - bumps of opposite direction were used to minimize them. In Pits 4 and 8, $\left|D_{y}\right|$ is less than $1.4 \mathrm{~mm}$, which at an rms momentum spread of $1.2 \times 10^{-3}$ results in an increase of the vertical beam size of the order of $3 \%$, which is negligible.

Different bunches meet the bunches of the train(s) in the opposite beam at the head-on collision points in IP4 and IP8, and at three different parasitic collision points in their vicinity. Hence, 
different bunches receive different beam-beam kicks, travel on different closed orbits, and all their orbital parameters are different. In particular, the simulated vertical orbit position $y$ and the vertical orbit slope $y^{\prime}$ at IP4 and IP8 are different for all four bunches in a train. The orbit difference between the highest and the lowest bunch is $\Delta y \approx 2.3 \mu \mathrm{m}$ whilst the nominal vertical beam radius is $\sigma_{y} \approx 8 \mu \mathrm{m}$. The difference between the highest and lowest slope is $\Delta y^{\prime} \approx 60 \mu \mathrm{rad}$ which is still small compared to the crossing angle $\varphi$ above which synchro-betatron resonances [8] are excited: $\varphi=\sigma_{y} / \sigma_{s} \approx 0.5 \mathrm{mrad}$, where $\sigma_{s} \approx$ $15 \mathrm{~mm}$ is the bunch length. The expected tune and chromaticity splits between the bunches for $0.3 \mathrm{~mA} /$ bunch are $\Delta Q_{x} \approx 0.003$, $\Delta Q_{y} \approx 0.005, \Delta Q_{x}^{\prime} \approx 0.01, \Delta Q_{y}^{\prime} \approx 2.0$. Both $\Delta Q_{x}$ and $\Delta Q_{y}$ should be small compared to the synchrotron tune $Q_{s} \approx 0.065$ in order to avoid synchro-betatron resonances. We attribute occasionally observed losses on individual bunches to the chromaticity splits.

\section{SINGLE BEAM EXPERIMENTS}

In order to obtain information on how the separator bumps for the bunch trains affect the beam behaviour, magnetic correctors were first used to excite bumps which follow as closely as possible the proposed electrostatic bumps. The required aperture and optical effects were studied. Bump amplitudes up to 2.7 times the nominal value did not affect the injection or the beam lifetime [9]. The reduced aperture at the beam dump in point 5 was also studied and with bunch train bumps of 1.8 times the nominal value no effects on the lifetime, the intensity and the background were observed [9]. Long bumps extending into the RF cavitites were successfully tested and no effects were observed on the beam. If the relative bump polarity is well chosen, the residual vertical dispersion can be minimized,During one experiment, the vertical rms dispersion was reduced from $48 \mathrm{~cm}$ to $20 \mathrm{~cm}$ by using alternating polarity.

The injection and accumulation in a single beam were tested by injecting one bunch of each train at a time. The maximum total intensity was obtained when bunches are filled in order, starting at the head of the train. The first injection of four trains of four bunches spaced by $87 \lambda_{\text {rf }}$ reached a total DC current of 5 $\mathrm{mA}$. After the installation of additional separators, further single beam studies were performed. With four trains of four bunches, $320 \mu \mathrm{A}$ per bunch were accumulated, limited by transverse mode coupling instabilities (c.f single bunch limit for one beam of $630 \mu \mathrm{A})$. The trailing bunches suffered more and saturated at lower levels. The losses were negligible when the beam was ramped to $45.6 \mathrm{GeV}$ and squeezed; the emittances were measured to be $\epsilon_{x}=30 \mathrm{~nm}$ and $\epsilon_{y}=3 \mathrm{~nm}$, and there were no noticeable variations amongst the bunches in a train. Longitudinal oscillations, larger for the trailing bunch, and a variation of the synchrotron tune along the trains were observed. These effects were predicted from the beam loading and RF modulation.

\section{HIGHER-ORDER MODE LOSSES}

The superconducting (SC) RF cavities are equipped with higher order mode couplers which can extract a few hundred watts of RF power which is sufficient for four or eight equidistant bunches in each beam. The damping due to HOM cou-

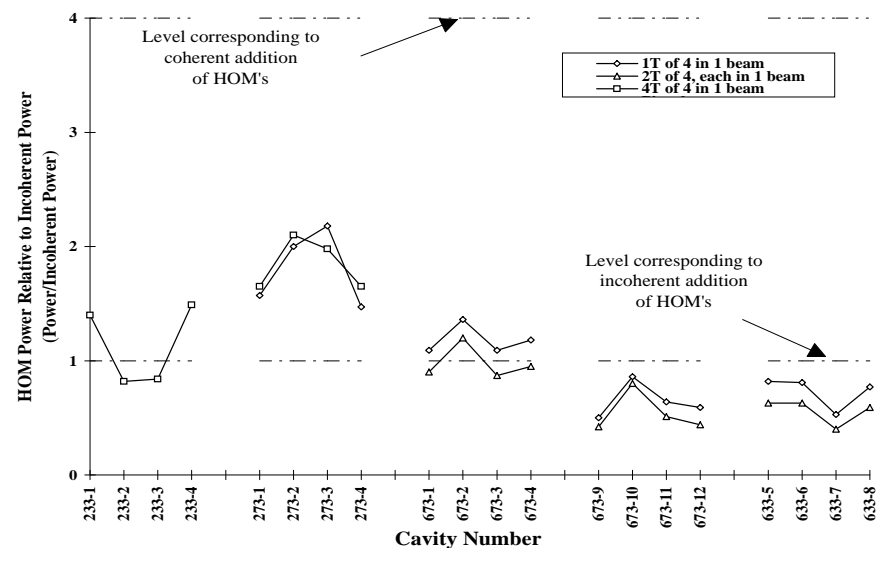

Figure. 2. HOM power measured on 20 cavities with different single beam bunch train configurations.

plers is, however, too small to damp the HOM losses between bunches in a train. Therefore the knowledge of the HOM losses and frequencies in each cavity becomes crucial since one can not exclude, by design, that any arbitrary bunch spacing is an exact multiple of the wavelength of a higher order mode. When this happens, the HOM fields of the $k$ bunches of a train must be added coherently, instead of simply adding their power (incoherent addition). The same argument holds for the counter rotating beams since the bunch trains in the $\mathrm{e}^{+}$and $\mathrm{e}^{-}$beams pass through the cavities too close in time for damping between passages.

The results of measurements of the HOM power on $20 \mathrm{SC} \mathrm{RF}$ cavities are shown in Figure 2; there is no indication of full positive coherent addition of fields. The HOM power is always about or even below the incoherent value, except for two cavities where the power corresponds to about twice that for simple addition. With the configuration installed in 1994, the effect of the two counter-rotating beams could not be checked because the trains crossed several kilometers away from the RF stations.

\section{COLLIDING BUNCH TRAINS}

With standard injection conditions the bunch current for one train of four bunches per beam was limited to around $300 \mu \mathrm{A}$. Following a low intensity trial during the setup period, the first attempt to establish colliding bunch trains successfully brought $279-247-221-230 \mu \mathrm{A}$ of positrons and $302-303-316-$ $281 \mu \mathrm{A}$ of electrons into collision. After appropriate orbit corrections the collimators were moved into position allowing ALEPH and DELPHI to take data. The lifetimes were good and the beam sizes comparable to normal 4 bunch operation.

Physics conditions were established three more times in the following three weeks [9] during which time the luminosity and background were optimised. Luminosity scans using the data for each collision within a train were performed. Figure 3 shows the luminosity for the crossings of bunches a, b, c and d during such a scan. The results confirmed the expected variation in vertical offsets caused by the beam-beam kicks from the parasitic encounters and both experiments gave consistent values for the luminosities of the order of $5-6 \times 10^{30} \mathrm{~cm}^{-2} \mathrm{~s}^{-1}$. 


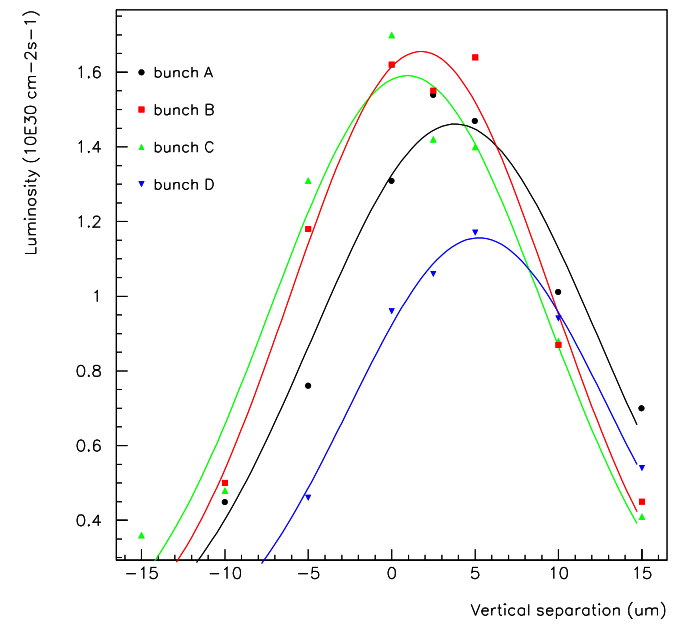

Figure. 3. Luminosity variation during a vertical separator scan in ALEPH.

The experiments had minimal problems and were able to fully explore their ability to take data with colliding bunch trains. Precise luminosity measurements were achieved for each bunch and measured online. Online tagging of the bunch number can also be obtained with high efficiency. The loss efficiency for the ALEPH hadron calorimeter and muon detector, a potential source of concern, appeared to be small and acceptable.

The combination of the vertical separation bump and powering the skew quadrupole magnets caused extremely large beam sizes $\left(\varepsilon_{x} \approx 100 \mathrm{~nm}\right.$ ) when the beams were brought into collision during physics operation with 4-on-4 bunches. The skew quadrupoles are inside the separation bumps and are used to compensate the solenoidal field of the experimental detectors. This effect was not observed with collisions only in points 4 and 8 during the tests, only when the bunch train bumps were on, the skew quadrupoles in 4 and 8 were powered and there were collisions in all 4 experiments. It was found that removing any one of these three conditions reduced the beam size to normal values.

\section{BACKGROUND}

Measurements with two colliding bunch trains [9] have shown no increase of the off-momentum background in the experimental detectors. However, a steep increase of the synchrotron radiation (SR) photon background has been observed in ALEPH and DELPHI for vertical bump amplitudes larger than $10 \mathrm{~mm}$ (Figure 4). Monte Carlo (MC) simulations confirm that this is due to photons, radiated from the peak of the bump. These photons strike the jaws of downstream vertical collimators and are back scattered into the detectors. For bump amplitudes below $10 \mathrm{~mm}$ most photons from the peak of the separator bump pass the interaction point without hitting downstream collimators and only a small increase of the photon background with amplitude is observed. The SR photon background with large separation bumps can be substantially reduced by superimposing asymmetric magnetic bumps in such a way that the amplitude of the incoming beam is reduced below the critical value of $10 \mathrm{~mm}$, while keeping the separation constant. The increased radiation of photons along the larger amplitude of the outgoing beam does not increase the

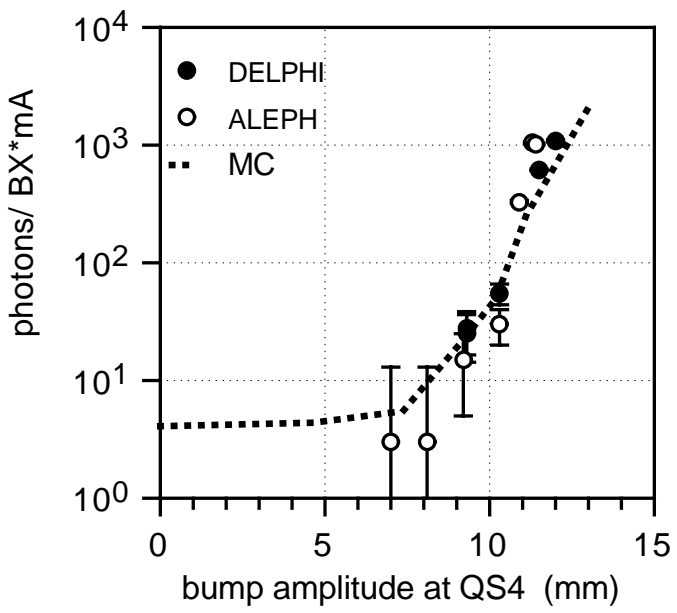

Figure. 4. Measured and simulated (MC) SR photon background in ALEPH and DELPHI as function of the separation.

SR rate, as these photons only strike the vacuum system a long way from the IP. The effect of the asymmetric correction bump has been experimentally verified [9].

\section{CONCLUSIONS}

The results of the tests in 1994 were judged encouraging enough for LEP to be adapted during the 1994/95 shutdown, for operation with four trains in each beam. Extra electrostatic separators were installed near IP2 and IP6, the separators near the odd pits were moved [6] and the skew quadrupole magnets were moved outside the separation bump. The application software and databases were modified and most of the other equipment groups also had to make changes to accommodate bunch train operation [9].

\section{References}

[1] R.E. Meller, 'Proposal for CESR Mini-B', CESR Operations Note CON 90-17 (1990).

[2] D. Rubin for CESR Operations Group, 'CESR Status and Plans', this conference.

[3] E. Keil, 'Lattice and Interaction Region Design for Z Factories', Proc. of the Joint US-CERN School, Benalmadena, Springer, 1994 - (Lecture Notes in Physics No. 425) 106126.

[4] R. Bailey et al., 'Studies of Bunch Trains in LEP', Proceedings of the 4th EPAC (1994) 445

[5] W. Herr, 'Bunch Trains without a Crossing Angle', in Proc. 4th Workshop on LEP Performance, ed. J. Poole, CERN SL/94-06 (DI) (1994) 323-332.

[6] B. Goddard et al., 'Modification of the LEP Electrostatic Separator Systems for Operation with Bunch Trains', this conference.

[7] T. Camporesi et al., 'Report from the Bunch Train Working Group', CERN/LEPC 94-13 (1994), CERN/LEPC 9416 (1994).

[8] A. Piwinski, 'Satellite Resonances due to Beam-beam Interaction', IEEE Trans. Nucl. Sci. NS-24 (1977) 1408.

[9] C. Bovet et al., 'Final Report of the 1994 Bunch Train Study Group', CERN/SL 94-95 (AP) (1994). 